\title{
Sequential effects in rudimentary auditory and visual tasks
}

\author{
PHILIP T. QUINLAN and NICHOLAS I. HILL \\ University of York, York, England
}

\begin{abstract}
Three experiments examined sequential effects in choice reaction time tasks. On each trial, a right/left positional judgment was made to a either a pure tone or a luminance increment in a visual array of box elements. In the first two experiments, a preparatory signal was presented prior to each imperative signal to indicate the relevant stimulus modality. At a short stimulus onset asynchrony (SOA) between the preparatory and the imperative signal (i.e., $60 \mathrm{msec}$ ), subjects were quicker to repeat the same response than to change their response when presented with successive tones, although no such repetition effect occurred on the visual target trials. Subjects were impaired if the stimulus modality changed across successive trials regardless of the modality of the target. At a longer SOA (i.e., $500 \mathrm{msec}$ ), these sequential effects were abolished; subjects were assumed to be able to prepare for the relevant modality because of the presentation of the preparatory signal. When the preparatory signals were omitted, in a final experiment, the modality-switching costs were still evident, but now inhibition of return occurred on both the auditory and the visual target trials-subjects were now impaired in responding when the target reappeared at its immediately previous location. It seems, therefore, that the repetition effect and modality-switching effects do dissociate. The data revealed clear differences between orienting attention to a particular spatial locale and focusing attention to a particular sensory modality.
\end{abstract}

The present paper reports on a series of experiments that examined sequential effects in choice reaction time (CRT) tasks. Central to this work is something that has been labeled the repetition effect (Rabbit, 1992)-an effect that is generally agreed to have its roots in the work of Bertelson (1961, 1965). As Rabbitt (1992, p. 313) stated, the repetition effect refers to the finding that "CRTs are significantly faster when the signals and responses that occur on successive trials are identical ... than when they are different ...." In exploring this finding, much of the early work used a single CRT task in which a small set of stimuli were divided into $n$ categories where each category demanded a particular keypress response. For example, Bertelson (1965) gave subjects a task in which the digits 2 and 4 were assigned to one keypress and the digits 3 and 5 to another. Of main interest were the possible effects that might arise across an adjacent pair of trials. For expository convenience, the first trial of any such pair (trial $n$ ) will be referred to as the prime trial, and the second member of each pair will be referred to as the target trial (trial $n+1)$.

Three sorts of pairs of trials were of interest: (1) identical trials, in which the stimuli on the prime and target trials were the same; (2) equivalent trials, in which the stimulus on the prime trial (henceforth the prime) and the stimulus on the target trial (henceforth the target) were

Correspondence should be addressed to P. T. Quinlan, Department of Psychology, University of York, Heslington, York, YO10 5DD, England (e-mail: ptq1@york.ac.uk). different but demanded the same response; and (3) different trials, in which the prime and target demanded different responses. It was reported that reaction times (RTs) were slow on different trials but were facilitated by the same amount on the identical and equivalent trials. Given these results, Bertelson (1965) argued that the repetition effect was located at the level of response mechanisms. This was because the same amount of facilitation arose in cases where the prime and target demanded the same response even if they were physically different.

As Pashler and Baylis (1991) have pointed out, though, subsequent work with similar tasks (e.g., Rabbitt, 1968; Smith, 1968) failed to endorse Bertelson's (1965) findings. They argued that this discrepant pattern of results can be understood on the assumption that the speeding to same-response stimuli occurs only when a preestablished mental category exists for the set of stimuli assigned to a particular response. Indeed, Pashler and Baylis examined these ideas in more detail in their experiments and went on to argue that the repetition effect was, essentially, stimulus specific. They considered two plausible accounts of the effect-namely, (1) a perceptual account, in which the identification of the particular stimulus is facilitated when it is re-presented, or (2) a response selection account. They favored the latter account and argued that the repetition effect was due to the formation of "shortcuts to response selection" (p. 43), with a particular stimulus directly invoking the appropriate response when it is repeated.

At one level, this "shortcut" account is nothing more than a restatement of Bertelson's (1965) original ac- 
count. He described subjects as adopting the method of sometimes bypassing a complete analysis of the stimulus in favor of simply repeating the last response if the current and immediately preceding stimuli matched each other. Indeed, Fletcher and Rabbitt (1978) went further and discussed the "bypass rule" as a more general description of subjects' performance. According to the bypass rule, subjects are predisposed to repeat the same response if the stimulus is the same across adjacent trials, and they will tend to change their response if the stimuli differ. Krueger and Shapiro (1981) provided further evidence showing the generality of the bypass rule in a number of different tasks and clearly demonstrated that subjects appeared to apply the rule even when successive stimuli mismatched on characteristics that were irrelevant to the currently adopted classification scheme. For instance, when subjects had to determine whether all of the displayed numerals were the same or not, a change in display size across successive trials affected performance.

In concluding their paper, Krueger and Shapiro (1981) suggested that "switching one's attention between trials has much in common with switching one's attention between modalities" (p. 261), as described by Posner (1978) and LaBerge (1973). The latter effects have more recently been described by Spence and Driver (1997) in terms of something they have called the modality-shifting effect (see, e.g., Hannes, Sutton, \& Zubin, 1968). The modality-shifting effect refers to the finding that RTs to targets are faster when the target and prime appear in the same modality on adjacent trials (ipsimodal trials) than when the target and prime appear in different modalities (crossmodal trials). Henceforth this effect will be referred to as the modality repetition effect. Krueger and Shapiro speculated that both the standard repetition effect and the modality repetition effect might have a common locus. They did not, however, examine this possibility in any more detail.

A possible difficulty in exploring these issues is that there is some concern over the very existence of the modality repetition effect because a number of studies have failed to reproduce it (at least with normal subjects: Bernstein, Pederson, \& Schurman, 1972; Kristofferson. 1967; Sutton, Hakerem, Zubin, \& Portnoy, 1961; Sutton \& Zubin, 1964). Moreover, in cases where the modality repetition effect has been found, problems exist over its interpretation. As Spence and Driver (1997) have pointed out, although many have postulated that the modality repetition effect may reflect the cost of having to switch attention between the sensory modalities (e.g., Kristofferson, 1967), a more mundane explanation may suffice. For instance, in cases where simple RTs were measured, subjects may have lowered their criterion for a response on ipsimodal relative to crossmodal trials (Spence \& Driver, 1997).

\section{The Present Study}

Given the uncertainty over the modality repetition effect, it seemed only reasonable to attempt a more thorough examination that avoids some of the pitfalls of earlier work. For example, the intention was to use CRT tasks, which would rule out any simple criterion account of performance. In addition, it appeared important to try to be clearer about the possible relationship between the modality repetition effect and the standard repetition effect as described by Bertelson $(1961,1965)$. To this end, tasks were designed in which the response and/or modality changed across the prime and the target trials.

In earlier pilot studies, subjects made high/low judgments about the pitch of a tone (henceforth auditory stimulus) and the position of a luminance increment in a visual array of box elements (henceforth visual stimulus). Subjects were provided with two response keys, with one key designated as the "high" key and the other as the "low" key. The pitch of the tone could be judged relative to an intermediate pitch tone that preceded the presentation of the tone stimulus. The position of the visual stimulus was judged relative to the horizontal midline of a visual display unit (VDU).

The results of this earlier work showed that (1) subjects were quickest to respond when both the exact stimulus and the response were repeated across adjacent trialsthat is, there was a stimulus-specific repetition effect; (2) subjects were also generally slower to respond when the stimulus modality changed between trials than when it remained the same-that is, there was an apparent modality repetition effect; (3) there was no effect of repeating the response when the stimulus modality of the target changed between trials; and (4) the pattern of differences was larger for the auditory than for the visual stimuli.

Overall these data provided evidence of both stimulusspecific repetition effects (as described by the short-cut account, Pashler \& Baylis, 1991) and, apparently, modalityswitching costs. A difficulty, though, in attempting to argue that the data revealed modality-switching costs is that different tasks were being carried out according to the modality of the stimulus. That is, subjects were judging the pitch of a tone and the position of a flash. On these grounds, it is, perhaps, more appropriate to claim that the data revealed task-switching costs (Rogers \& Monsell, 1995) rather than modality-switching costs per se. The costs may well have reflected difficulties in something Rogers and Monsell termed task-reconfiguration. In an attempt to examine whether modality-switching costs exist in the absence of having to switch tasks across trials, the present experiments used the same location judgment for both auditory and visual stimuli. The basic design of the tasks was such that effects of stimulus, response, and modality repetition could be examined in concert. Indeed, a primary aim was to examine the relationship between the standard repetition effect and the modality repetition effect.

The experiments also addressed the issue of whether or not attentional mechanisms are implicated in the sequential effects described. In this respect, the pilot data were instructive in revealing that subjects demonstrated 
larger effects when they made responses to the auditory stimuli than to the visual stimuli: In fact the standard repetition effect was of only marginal significance when subjects made responses to the visual stimuli. This contrasting pattern of results was taken to indicate differences in the allocation of attention to the auditory and visual modalities, as described in the visual dominance account put forward by Posner, Nissen, and Klein (1976). By this account, it seemed that subjects in the pilot study exhibited an attentional bias toward the visual modality and that on visual trials, each of the response outcomes was given an equal weighting on each trial. Neither response was favored; hence, neither response was facilitated by the presence of an identical stimulus on the previous trial. In contrast, when the attentional bias did not operate toward the modality of the stimulus (i.e., on the auditory trials), it seemed that subjects performance conformed to the bypass rule. The plausibility of this account is tested further in the present experiments.

In summary, this paper reports on three experiments. In each of the experiments, subjects made left/right locational judgments to either a visual or an auditory imperative signal. In the first two experiments, on every trial the imperative signal was preceded by a preparatory signal that informed of the modality, but not the location, of the imperative signal. Experiment 1 was run as a partial replication of one of the earlier pilot studies; a basic aim was to examine whether similar patterns of performance now arose when the same task was carried out for both the auditory and the visual stimuli. In Experiment 1, the stimulus onset asynchrony (SOA) between the preparatory and the imperative signals was set at $50 \mathrm{msec}$, and it was of some further interest to explore whether performance would be unchanged when this SOA was lengthened. To this end, Experiment $2 \mathrm{~A}$ was run, in which the SOA was set at $500 \mathrm{msec}$. It was thought that, given such a lag, subjects might be able to operate most effectively on the basis of the information contained in the preparatory signal and focus their attention on the modality of the ensuing imperative signal. As a consequence, such experimental conditions should reflect the degree to which task performance was under subjective (attentional) control.

In a final bid to examine the notion that task performance does reveal the operation of attentional mechanisms, Experiment $2 \mathrm{~B}$ was carried out. Now the preparatory signals were omitted altogether; otherwise, the same timing parameters as those in Experiment $2 \mathrm{~A}$ were used. Here the aim was to examine the degree to which performance would be affected by the presence of the preparatory signal rather than by the amount of time that subjects had to prepare for the presentation of the imperative signal.

\section{GENERAL METHOD}

The same general testing procedures and equipment were used throughout. Where there are particular differences across the experiments, these details will be conveyed at the appropriate stage.

\section{Apparatus}

All displays were presented on a 14-in. Acorn Color monitor. The monitor was at a fixed distance from a chinrest. When a subject sat at the chinrest, the viewing distance from the screen was $57 \mathrm{~cm}$. The chinrest was adjusted for each subject in such a way that the center of the screen was at eye level. The timing, stimulus presentation, and data collection were under the control of an Acorn Archimedes R260 computer. Two finger response keys were provided and interfaced with the computer. Tones were presented via a pair of Sennheiser HD 56 earphones. All testing was carried out in a small testing room, and all of the equipment except for the computer was housed in this room.

\section{Stimuli and Design}

The basic visual stimulus consisted of a $3 \times 3$ array of small square boxes (after Posner $\&$ Cohen, 1984, p. 535). Each box measured approximately $1^{\circ} \times 1^{\circ}$ of visual angle. Due to screen distortion, the horizontal center-to-center distance of the boxes was $5.0^{\circ}$ of visual angle, but the vertical center-to-center distance was $4.5^{\circ}$ of visual angle. All of the subjects were run with the lights off in a small testing room. Under these conditions, the background luminance of the VDU screen was $0.11 \mathrm{~cd} / \mathrm{m}^{2}$. The luminance of the contour of an individual box was $0.50 \mathrm{~cd} / \mathrm{m}^{2}$; this increased to $2.00 \mathrm{~cd} / \mathrm{m}^{2}$ when the box acted as a preparatory signal or a target. When a visual stimulus was presented (on a visual trial), a visual preparatory signal preceded the presentation of the visual imperative signal. The preparatory signal in this case was the brightening of the two outer boxes in the middle row.

The auditory stimuli used were pure tones. The tones were produced digitally (8-bit quantization) with a sampling rate of $41.7 \mathrm{kHz}$. When an auditory stimulus was presented (on an auditory trial), a binaural tone acted as a preparatory signal. The frequency of the tones was $488 \mathrm{~Hz}$. Stimulus intensities gave peak sound pressure levels of $86 \mathrm{~dB}(\mathrm{~A})$ at the headphones, calibrated with a Brüel and Kjaer artificial ear- and sound-level meter.

In generating the experimental trials, the starting point was to define a basic pair of trials. The first member of the pair (trial $n$ ) was known as the prime trial and the second member of the pair (trial $n+1$ ) was known as the target trial. Each trial contained a preparatory signal followed by an imperative signal. On a visual trial, subjects responded to the visual imperative signal, and on an auditory trial, subjects responded to the auditory imperative signal. Both the visual and the auditory tasks demanded a binary judgment of the location of the imperative signal.

In each experiment, 16 basic pairs of trials were generated. This set of 16 corresponded to the factorial combinations of prime type (auditory vs. visual), target type (auditory vs. visual), response type (same as prime vs. different from prime), and response identity (left vs. right). Random orders of these 16 pairs of trials gave rise to the set of trials used in the individual experiments. Subjects were naive as to the pairing of trials, and there was nothing in the experiment to signify the nature of the prime trial/target trial relationship.

In this experiment, subjects judged the (left vs. right) position of the visual imperative signal and the (left vs. right) position of the auditory imperative signal. On a visual trial, the preparatory signal (the brightening of the outer boxes in the middle row of the array) was followed by the brightening of the two corner boxes in the left column (designated as the left visual stimulus) or the brightening of the two corner boxes in the right column (designated as the right visual stimulus). Here the term corner refers to the top-most and bottom-most boxes in the two outer columns. On an auditory trial, the binaural tone preparatory signal was followed either by a monaural tone in the left ear (the left auditory stimulus) or a monaural tone in the right ear (the right auditory stimulus). The influence of the preparatory signals is examined in much more detail later in the paper. 
Table 1

Summary Statistics (Mean Reaction Times [RT], in Milliseconds, Standard Deviations $[S D]$, and Percentage of Error [PE]) for Performance on the Target Trials in Experiment 1

\begin{tabular}{|c|c|c|c|c|c|c|}
\hline \multirow{3}{*}{$\begin{array}{l}\text { Modality } \\
\text { Repetition }\end{array}$} & \multicolumn{6}{|c|}{ Response Repetition } \\
\hline & \multicolumn{3}{|c|}{ Present } & \multicolumn{3}{|c|}{ Absent } \\
\hline & RT & $S D$ & $\mathrm{PE}$ & RT & $S D$ & $\overline{P E}$ \\
\hline \multicolumn{7}{|c|}{ Auditory } \\
\hline Present (auditory) & 318 & 50 & 1.0 & 347 & 57 & 3.1 \\
\hline Absent (visual) & 358 & 57 & 0.4 & 371 & 66 & 2.5 \\
\hline \multicolumn{7}{|c|}{ Visual } \\
\hline Present (visual) & 337 & 39 & 3.3 & 331 & 38 & 1.6 \\
\hline Absent (auditory) & 350 & 40 & 2.3 & 350 & 50 & 0.8 \\
\hline
\end{tabular}

All subjects responded with a right keypress whenever an imperative signal occurred in the right-hand space, and they responded with a left keypress whenever an imperative signal occurred in the left-hand space. Given this design, the response identity was either "right" or "left" for both the auditory and the visual tasks. ${ }^{1}$

\section{EXPERIMENT 1}

Given the task confounds that were present in the earlier pilot experiments, the first experiment to be reported here examined performance in interleaved visual and auditory tasks in which subjects carried out the same locational judgment on the visual and auditory signals. The basic idea was that such tasks might provide evidence for modality-switching costs, in the absence of any possible task-switching costs, and, in addition, perhaps, also reveal how these relate to the standard repetition effect.

\section{Method}

Subjects. Twelve subjects selected from the Department of Psychology subject panel participated in the experiment. Eight psychology student subjects received course credit for participation. Four other students were paid $£ 3$ per session. All reported having normal or corrected-to-normal vision and none reported any known hearing problems.

Procedure. Each subject was tested individually. Prior to the experiment, the subjects were given verbal instructions about the task constraints. The nature of each trial was explained in detail. On a visual trial, the sequence of events was as follows. Initially a central fixation plus sign was presented in the center box for $500 \mathrm{msec}$. The plus sign was $0.3^{\circ}$ (high) $\times 0.4^{\circ}$ (wide) of visual angle (luminance $=2.00 \mathrm{~cd} / \mathrm{m}^{2}$ ). The fixation plus sign was immediately replaced by the preparatory signal for $60 \mathrm{msec}$. The imperative signal was presented at the offset of the preparatory signal for $140 \mathrm{msec}$ and subjects had up to $3.000 \mathrm{msec}$ to respond. When the computer detected a response or the response deadline had expired, contingent feedback was presented on the screen. The word "correct" or "error" was presented slightly below the position of the overall array of boxes. This lasted for $500 \mathrm{msec}$, and if a mistake had been detected, then an additional $400 \mathrm{msec}$ was added to this interval. Next the screen went blank for $500 \mathrm{msec}$. Therefore, the intertrial interval (ITI) was set at $1,000 \mathrm{msec}$ after a correct response and 1,400 msec after an incorrect response.

On an auditory trial, the sequence of events was the same except that in the place of the visual preparatory signal and imperative signal, an auditory preparatory signal and imperative signal were pre- sented. As before, at the start of a trial the array of boxes was presented on the screen and the same fixation cross was presented. However, now the appearance of the boxes remained unaltered and the subject was presented with the tones over the earphones. Given the equipment constraints, the duration of the preparatory signal was $50 \mathrm{msec}$ and the duration of the target was $150 \mathrm{msec}$. The target was presented at the immediate offset of the preparatory signal. These values were as near as they could be to the timings used with the visual stimuli. The same visual feedback was presented at the end of the trial as had occurred on the visual trials.

Following the verbal instructions, subjects were told that responses would be timed and that they should try to respond quickly without making too many errors. The experimenter then left the room and the subject worked through a single block of 32 practice trials. This block of trials contained a random order of the basic 16 pairs of trials. Following this practice block, subjects were informed that the experimental trials were divided into five further blocks and that a message would be presented on the screen when a block had finished. To initiate the next block, they were instructed to press a foot switch and to defer this if they wanted to rest. Although subjects were not informed, each experimental block of trials contained 128 individual trials. Each experimental block contained eight random orders of the 16 basic pairs of trials. Across the five blocks of trials, subjects were presented with 640 experimental trials in total (i.e., 320 prime -target pairs). Overall, a given testing session lasted approximately $40 \mathrm{~min}$.

\section{Results}

In order to simplify discussion of the results, emphasis is placed on analyses in which averaging was carried out over left and right responses. When actual response type was entered as an additional factor and produced any statistically significant effects, these are discussed accordingly.

Of primary interest was performance on target trials. Initially, mean RTs were computed only over target trials, and in order for a trial's score to be included, the response on both the prime trial and the target trial needed to have been correct. The correctness of the response on the prime trial having been taken into account, the prime trial data were not analyzed further. Table 1 shows the summary statistics of these data together with the summaries of the errors scored on target trials.

The RT data from the target trials were entered into a $2 \times 2 \times 2$ repeated measures analysis of variance (ANOVA). Here the factors were target modality (auditory vs. visual), modality repetition (present vs. absent), and response repetition (present vs. absent). This analysis revealed that the only statistically significant main effect was that of modality repetition $[F(1,11)=27.17, p<.001$; $F<1.0$ for the main effect of both target modality and response type]. Generally speaking, subjects were faster in making their responses if the modality of the stimulus was repeated than when it was not, that is, there was an overall modality repetition effect. In addition, all of the interactions, except for the modality repetition $\times$ response repetition interaction $(F<1.0)$, were statistically significant $[F(1,11)=6.05, p<.05$, for the target modality $\times$ modality repetition interaction; $F(1,11)=16.53$, $p<.01$, for the target modality $\times$ response repetition interaction; and $F(1,11)=6.79, p<.05$, for the target 
modality $\times$ modality repetition $\times$ response repetition interaction]. The two-way interactions reflect the fact that response repetition reduced RT only for auditory targets, and not for visual targets, and that modality repetition reduced RT much more for auditory targets than for visual targets. The three-way interaction reflects the fact that for auditory targets, but not for visual targets, subjects seemingly relied, in part, on the bypass rule (i.e., repeat the response if the stimulus or some aspect thereof, e.g., modality, is repeated from the preceding trial). Response repetition helped in general on auditory target trials, but especially helped when the modality also was repeated, as the bypass rule would predict.

To analyze this pattern of results further, two separate $2 \times 2$ repeated measures ANOVAs were carried out on the data for the two target types (auditory vs. visual). The analysis of the data from the auditory target trials revealed statistically significant main effects of both modality repetition $[F(1,11)=22.67, p<.001]$ and response repetition $[F(1,11)=11.13, p<.01]$. The modality repetition $\times$ response repetition interaction was also statistically reliable $[F(1,11)=5.48, p<.01]$.

The interaction was further decomposed using a Tukey's HSD test. Responses were generally faster when the prime and target were presented in the same modality than when there was a switch in modality across the prime and the target trials. The only difference here not to reach statistical significance was between the case where the response was maintained but the modality changed and the case where the response was changed and the modality remained the same (358 vs. $347 \mathrm{msec}$; see Table 1; $p>.05$ ). Most importantly, though, whereas there was an effect of response repetition when the prime and target were both auditory stimuli $(p<.01)$, there was no effect of response repetition when the prime was a visual stimulus and the target was an auditory stimulus $(p>.05)$. When the stimuli were both auditory, same responses were faster than different responses.

The analysis of the data from the visual target trials revealed only a statistically significant main effect of prime modality $[F(1,11)=13.83, p<.01]$. Neither the main effect of response repetition nor the modality repetition $X$ response repetition interaction was statistically reliable $[F<1.0]$. Overall, subjects were slower to respond to the visual targets when the modality changed across trials than when it remained the same. There was no response repetition effect in these data.

Errors were collated for all target trials. Summary statistics of these data are included in Table 1 and, as can be seen, error rates were low (i.e., mean error rates over subjects were less than $4 \%$ in any condition). Initially, these data were expressed as a mean proportion incorrect for each subject/condition, and then these proportions were arcsine transformed following the guidelines set out by Winer (1971). These transformed data were entered in the same sort of ANOVA as that used with the RT data. This analysis revealed that only the target modality $\times$ response repetition interaction was statistically reliable $[F(1,11)=$
$10.37, p<.01 ; F(1,11)=2.75, p>.05$, for the main effect of modality repetition; $F<1.0$, all other tests]. The target modality $\times$ response repetition interaction was further examined using an HSD test but this failed to reveal any statistical differences between the various means. The pattern of differences, though, indicated that whereas subjects were more accurate in repeating the same response than changing responses on auditory target trials, the reverse effect obtained on the visual target trials.

When actual response type was included as a factor in the analyses, only one further statistically significant effect emerged. In the analysis of the RT data, there was a statistically significant interaction between response type and response repetition $[F(1,11)=6.98, p<.05]$. Unfortunately, further statistical examination of this interaction failed to reveal its cause (all pair-wise comparisons, $p>.05$, HSD test). Visual inspection of the means, though, indicated that whereas subjects showed a large repetition benefit when making a left response ( 359 vs. $331 \mathrm{msec}$, a mean difference of $28 \mathrm{msec}$ ), no such benefit accrued when subjects made a right response ( $340 \mathrm{vs} .351 \mathrm{msec}$, a mean difference of $-11 \mathrm{msec}$ ). There is no obvious reason for this result. No further statistically significant effects of response type were found in the analyses of the $\mathrm{RT}$ or error data.

\section{Discussion}

Overall, the data were in keeping with what was expected on the basis of the pilot work. Of particular note, though, is the finding that subjects were generally slower to respond when the stimulus modality changed between trials than when it remained the same. Given that the same task was performed for both the auditory and the visual stimuli, this pattern of differences suggests that there was a true cost in having to switch attention between the different modalities. The present data clearly indicate modality-switching costs, and such costs were present in the data for both the auditory and the visual stimuli.

Nevertheless, at a more detailed level, there was clear evidence for a contrasting pattern of performance with the two types of target. When subjects made responses to the visual targets, the only effect to emerge in the RT data was the modality-switching cost. However, when subjects made responses to auditory targets, modality repetition reduced RT, and the speeding was especially evident on trials in which both the response and the modality were repeated, which indicates that in responding to the auditory targets, subjects' performance conformed to the bypass rule as discussed by Krueger and Shapiro (1981). Not only were subjects faster to repeat their response to the same auditory stimulus over adjacent trials than when they had to change their response, but they were also more accurate on the repeated trials than on the different response trials. These data suggest, therefore, that subjects had a tendency to repeat their previous responses when confronted with an auditory target. Interestingly, the reverse effect was found in the 
visual target data. Subjects tended to be more accurate in making a different response on visual target trials than when they had to repeat the immediately prior response.

Overall, this contrasting pattern of results on the auditory and visual target trials replicates that found in the pilot studies. The observed pattern of effects is consistent with the idea that subjects exhibit a general bias toward the visual modality inasmuch as they were able to, essentially, overcome any deleterious effects of having to change their responses across adjacent trials. If anything, they exhibited a slight tendency to be more accurate in responding to a visual target if a response change was called for. Most importantly, though, the contrast between performance on the auditory and the visual target trials suggests that the standard repetition effect and the modality repetition effect are independent. Whereas the modality repetition effect occurred on both types of target trials, the standard repetition effect arose only on the auditory target trials. Further discussion of this point is included below.

\section{EXPERIMENTS 2A AND 2B}

In an attempt to further examine the degree to which the different sorts of sequential effects reported are influenced by attentional factors, two additional experiments were carried out. In Experiment $2 \mathrm{~A}$, a pause was introduced between the offset of the preparatory signal and the onset of the imperative signal. Subjects again made left versus right decisions about the auditory and visual stimuli, but now they had approximately half a second to prepare for the modality of the imperative signal. The rationale for this manipulation was that if the reported modality repetition effect does reflect an attentional component, then it should either be diminished or even abolished if subjects are given enough time to prepare for the modality of the imperative signal. Alternatively, if attention plays no role, then performance ought to be, essentially, the same as that described in Experiment 1.

An issue that still remained, though, was the degree to which performance in that task was affected primarily by the presence of the preparatory signal rather than the introduction of the lag between the onset of the trial and the presentation of the target. In this respect, two possibilities suggested themselves. The first was that subjects were able to operate on the basis of the information conveyed by the preparatory signal and consequently focus their attention on the relevant modality. The second possibility was that performance in that task might merely reflect the fact that subjects had more time to prepare for the imperative signal after the presentation of the fixation cross. According to this second account, the presence of the preparatory signal was irrelevant.

In an attempt to test these two alternatives, Experiment $2 \mathrm{~B}$ was carried out, in which subjects made the same locational judgments as before but now no preparatory signals were presented. The same timing parameters as those used in Experiment 2A were used, but no preparatory signal was presented between the fixation cross and the imperative signal. Under these conditions, the responsestimulus interval (RSI) was kept the same as before, but now subjects never knew whether an auditory or a visual stimulus would occur as the imperative signal. If it is merely the preparation interval that is critical, then performance should be the same across Experiments $2 \mathrm{~A}$ and 2B. In contrast, if the lack of sequential effects in the data was due to subjects' being able to prepare for the relevant modality because of the preparatory signal, then a different pattern of performance should obtain when the preparatory signals are omitted.

\section{Method}

The same locational judgment tasks and experimental setup were used in Experiments $2 \mathrm{~A}$ and $2 \mathrm{~B}$ as before. Experiment $2 \mathrm{~A}$ was a replication of Experiment 1 apart from the fact that now the SOA between the preparatory signal and the imperative signal was increased from 60 to $500 \mathrm{msec}^{2}$ The same timing parameters were used in Experiment 2B as in Experiment 2A, but now there never was a preparatory signal on any trial.

Subjects. Two separate groups of 10 subjects were selected from the Department of Psychology subject panel to participate in each experiment. Six psychology student subjects in each group received course credit for participation. Four students in each sample were paid $£ 3$ per session. All reported having normal or corrected-tonormal vision and none reported any known hearing problems.

\section{Results}

Experiment 2A. Table 2 shows the summary statistics of these data together with the summaries of the errors scored on target trials. The RT data were analyzed as before, and this analysis revealed that only the main effect of target type (i.e., faster responses to auditory targets than to visual targets) reached statistical significance $[F(1,9)=$ $7.22, p<.05 ; F(1,9)=2.34, p>.05$, for the main effect of response repetition; and $F<1.0$ for all other tests in the analysis]. A similar analysis of the transformed error data revealed that none of the tests produced a statistically significant result (all $p \mathrm{~s}>.05$ ). On the average, less than $3 \%$ errors were made in any one condition.

When response type was entered as an additional factor in the analysis of the RTs, no further statistically significant effects arose. A comparable analysis of the error data was precluded by the fact that in certain cells in the design, none of the subjects committed an error.

Experiment 2B. Table 3 shows the summary statistics of these data together with the summaries of the actual errors scored on target trials. The RT data were analyzed as before. This analysis revealed that only the main effects of modality repetition $[F(1,9)=27.13, p<$ $.01]$ and response repetition $[F(1,9)=7.01, p<.05]$ were statistically significant $[F(1,9)=1.85, p>.05$, for the main effect of target modality; $F(1,9)=2.21, p>.05$, for the modality repetition $X$ response repetition interaction; $F(1,9)=3.25, p>.05$, for the target modality $\times$ response repetition interaction; $F<1.0$, all other cases]. This pattern of results showed that subjects were slower to respond when the modality of the target changed across 
Table 2

Summary Statistics (Mean Reaction Times [RT], in Milliseconds, Standard Deviations [SD], and Percentage of Error [PE]) for Performance on the Target Trials in Experiment 2A

\begin{tabular}{|c|c|c|c|c|c|c|}
\hline \multirow{3}{*}{$\begin{array}{l}\text { Modality } \\
\text { Repetition }\end{array}$} & \multicolumn{6}{|c|}{ Response Repetition } \\
\hline & \multicolumn{3}{|c|}{ Present } & \multicolumn{3}{|c|}{ Absent } \\
\hline & RT & $S D$ & PE & RT & $S D$ & PE \\
\hline \multicolumn{7}{|c|}{ Auditory } \\
\hline Present (auditory) & 308 & 49 & 2.8 & 320 & 71 & 1.3 \\
\hline Absent (visual) & 310 & 49 & 0.5 & 329 & 61 & 1.5 \\
\hline \multicolumn{7}{|c|}{ Visual } \\
\hline Present (visual) & 335 & 73 & 0.8 & 340 & 72 & 0.8 \\
\hline Absent (auditory) & 337 & 62 & 1.3 & 341 & 75 & 1.0 \\
\hline
\end{tabular}

trials than when the modality remained unchanged. In addition, subjects were slower to repeat the same responses across trials than when they had to change their responses.

The accuracy data were analyzed in the same manner; however, the only test to reveal a statistically significant result was of the main effect of target modality $[F(1,9)=$ $9.92, p<.01 ; F(1,9)=2.32, p>.05$, for the target modality $\times$ modality repetition interaction; $F(1,9)=$ $1.50, p>.05$, for the modality repetition $\times$ response repetition interaction, $F<1.0$, all other cases]. In summary, the analysis revealed that subjects made more errors when responding to the auditory target than they did when responding to the visual target. Again, error rates were low, with no condition mean exceeding $6 \%$.

When response type was entered as an additional factor in the analysis of the RTs, the only further statistically significant result pertained to the main effect of response type $[F(1,9)=30.00, p<.001]$. For whatever reason, subjects were faster in making a left than a right response ( $315 \mathrm{vs} .321 \mathrm{msec}$; a mean RT difference of $5 \mathrm{msec}$ ). A comparable analysis of the error data was precluded by the fact that in certain cells in the design, none of the subjects committed an error.

A final set of analyses was carried out to compare performance across Experiments $2 \mathrm{~A}$ and $2 \mathrm{~B}$. Here the same three within-subjects factors of target modality, modality repetition, and response repetition were used; in addition, experiment ( $2 \mathrm{~A}$ vs. $2 \mathrm{~B}$ ) was entered as a between-groups factor. Analysis of the RTs revealed statistically significant main effects of target modality (i.e., faster responses to auditory targets than to visual targets) $[F(1,18)=7.33$, $p<.05]$ and modality repetition $[F(1,18)=24.45, p=$ $.001 ; F<1.0$ for both the main effects of experiment and response repetition]. In addition, both the experiment $X$ modality repetition interaction $[F(1,18)=15.42, p=.001]$ and the experiment $\times$ response repetition $[F(1,18)=7.65$, $p<.05]$ reached statistical significance. The experiment $x$ modality repetition interaction reflected the fact that whereas there was no modality repetition effect in the data for the Experiment $2 \mathrm{~A}$ subjects, there was such an effect in the data for the Experiment $2 \mathrm{~B}$ subjects. In addition, the experiment $X$ response repetition interaction revealed that whereas there was no response repetition effect in the data for the Experiment $2 \mathrm{~A}$ subjects, there was an effect in the data for Experiment $2 \mathrm{~B}$ subjects. Subjects in Experiment $2 \mathrm{~B}$ were impeded in responding when the target stimulus was re-presented at the prime's location.

A comparable analysis of the transformed error data merely revealed a statistically significant main effect of target modality $[F(1,18)=12.08, p<.01]$. Overall, subjects were less accurate in responding to the auditory target than to the visual target. When one takes into account both the RT and error data, it seems that there was evidence for a speed-error tradeoff. Although overall subjects' RTs were faster to the auditory than to the visual targets, responses to the auditory targets were also less accurate than those to the visual targets.

\section{Discussion}

The data from Experiment $2 \mathrm{~A}$ stand in contrast to those of Experiment 1: The only statistically significant effect revealed that overall subjects were slower to make responses to the visual than to the auditory targets. More importantly, though, the lack of any sequential effects in that data is consistent with the idea that subjects were able to effectively operate on the basis of the preparatory signal. Indeed, since the same classification scheme was used in both the visual and the auditory tasks, subjects were able to prepare for the modality of the imperative signal. Any costs associated with switching between modalities, as seen in the data for Experiment 1, were abolished when subjects were so prepared. Given this, further weight is added to the claim that the modalityswitching costs do reflect the operation of an attentional mechanism: When subjects could prepare for the modality of the imperative signal, performance on the previous trial had no influence.

In addition, the data from Experiment $2 \mathrm{~B}$ rule out the idea that performance in Experiment 2A merely reflected the fact that subjects were given sufficient time to prepare for the imperative signal after the offset of the fixation cross. Experiment $2 \mathrm{~B}$ replicated Experiment $2 \mathrm{~A}$ in all respects except that now the previous preparatory sig-

Table 3

Summary Statistics (Mean Reaction Times [RT], in Milliseconds, Standard Deviations $[S D]$, and Percentage of Error [PE]) for Performance on the Target Trials in Experiment 2B

\begin{tabular}{|c|c|c|c|c|c|c|}
\hline \multirow{3}{*}{$\begin{array}{l}\text { Modality } \\
\text { Repetition }\end{array}$} & \multicolumn{6}{|c|}{ Response Repetition } \\
\hline & \multicolumn{3}{|c|}{ Present } & \multicolumn{3}{|c|}{ Absent } \\
\hline & RT & $S D$ & PE & RT & $S D$ & $\mathrm{PE}$ \\
\hline \multicolumn{7}{|c|}{ Auditory } \\
\hline Present (auditory) & 297 & 36 & 3.1 & 296 & 42 & 2.7 \\
\hline Absent (visual) & 329 & 55 & 4.6 & 323 & 69 & 3.5 \\
\hline \multicolumn{7}{|c|}{ Visual } \\
\hline Present (visual) & 315 & 36 & 1.3 & 301 & 36 & 2.7 \\
\hline Absent (auditory) & 357 & 48 & 0.6 & 330 & 52 & 1.9 \\
\hline
\end{tabular}


nals were no longer included. That is, whereas before the presentation of the preparatory signal allowed subjects to prepare for the relevant modality, now subjects were unable to do so because no preparatory signal was presented. Under these conditions a quite different pattern of effects emerged. The data were clear in showing (1) a strong modality-switching cost (i.e., overall, subjects were slower to respond when the stimulus modality changed across trials than when it remained the same), and (2) subjects were now slower to repeat the same response over trials than to make the alternative response. The presence of both of these effects offers further support that the subjects were able to operate on the basis of the information provided by the preparatory signals used in Experiment 2A. By implication, these data suggest that the original sequential effects reported in Experiment 1 (and in the previous pilot work) were critically linked to the presence of the preparatory signals used: In the absence of the preparatory signals, quite different sequential effects obtained. Further discussion of these points is included below.

\section{GENERAL DISCUSSION}

In summary, three experiments have been reported that examined the nature of sequential effects in CRT. Although there is an extensive literature on how response repetition effects relate to the nature of the repeated stimulus characteristics (see Rabbitt, 1992), an aim here was to examine these effects when the sequentially presented stimuli both occurred in the same sensory modality as opposed to when the modality changed over adjacent trials. In all cases, the same positional judgment was carried out on the stimuli presented in the different modalities.

\section{The Standard Repetition Effect and the Modality Repetition Effect}

In Experiment 1, a short preparatory signal preceded each imperative signal, and there was a significant response repetition benefit in the data for the auditory target trials. Indeed, there was more general evidence that subjects were operating according to the bypass rule (i.e., repeat the response if the stimulus or some aspect thereof, e.g., modality, is repeated from the preceding trial) (Fletcher \& Rabbitt, 1978; Krueger \& Shapiro, 1981) when presented with the auditory stimuli. Not only were subjects quickest in correctly repeating their previous responses, but they also exhibited a tendency to repeat the previous response when primed with a same-modality stimulus even if two different tones were presented on successive trials. A quite different pattern of performance occurred on the visual target trials. Here the only statistically significant effect in the RT data was the modality repetition effect: Subjects were slower in responding when the modality changed across trials than when it remained the same. In addition, subjects tended to be more accurate when they had to change their responses than when they could repeat their responses. The RT data replicated the effects revealed in the previous pilot studiesnamely, that subjects' performance was essentially unaffected by the immediately previous response when visual targets were presented. On these grounds, it was assumed that the lack of any sequential effects reflected an attentional bias to the visual modality in such a way that all response outcomes were equally weighted. The present accuracy data qualify this conclusion somewhat because subjects' accuracy did suffer if a visual target occurred in the same side of space as the previous stimulus. The significance of this effect is discussed in more detail below.

Of some additional import was the finding that modality-switching costs occurred on both auditory and visual target trials. The presence of the modality repetition effect in the absence of the standard repetition effect on the visual target trials therefore provided firm evidence that the two effects dissociate and hence appear to reflect the operation of different underlying mechanisms. Moreover, since the same task was undertaken regardless of stimulus modality, these performance costs could not be confused with task-switching effects (Rogers \& Monsell, 1995). It seems, therefore, that the modalityswitching costs described here reflect a processing penalty in having to switch attention between the two modalities (Kristofferson, 1967; LaBerge, 1973). The standard repetition effect therefore reflects different processes from those responsible for directing attention to a particular modality.

In Experiment 2A, the lag between the preparatory signal and the imperative signal on each trial was increased even though the RSI was similar to that used previously. Under these conditions, no sequential effects were observed. Initially, these data seemed to fit most comfortably with the idea that subjects had been able to operate on the basis of the information provided by the preparatory signal and to attend fully to the relevant modality. Against this, though, it was argued that the null effects arose simply because the subjects had more time to prepare for the arrival of the imperative signal. To test this idea, Experiment 2B was carried out, in which the same timing parameters were used as in Experiment $2 \mathrm{~A}$, but now no preparatory signals were presented. Under these conditions, a contrasting pattern of effects emerged. Now the previously described modality-switching effect was found, but a quite different sequential effect was also present in the data, different from the standard repetition effect: Subjects were now slower to repeat the same response than they were to make the alternative response.

\section{Inhibition of Return}

This particular sequential effect was unexpected given the pattern of effects reported in Experiment 1 . However, it is not completely surprising given the growing literature on inhibition of return (Reuter-Lorenz, Jha, \& Rosenquist, 1996; Tanaka \& Shimojo, 1996; Terry, Valdes, \& Neill, 1994). Although there are many subtle variations, 
the basic inhibition of return effect was originally shown in a visual cuing paradigm (Posner \& Cohen, 1984). The same sort of box displays were used there as were used here, except only a single row of three boxes was presented. A trial began with the brightening of one of the peripheral boxes, and subjects had to respond to the onset of a following target (the brightening of a small central region within one of the three boxes). When the cue-target SOA was relatively short (i.e., $100 \mathrm{msec}$ or less), subjects were facilitated in responding if the cue and target appeared at the same location. However, at longer SOAs (i.e., greater than $300 \mathrm{msec}$ ), subjects were impeded in responding if the cue and the target fell at the same location. The latter type of response slowing has come to be known as inhibition of return. Although this effect is well established in visual tasks, the present data show that inhibition of return is also manifest when subjects made consecutive judgments about the location of auditory signals (see also Reuter-Lorenz et al., 1996). In the present context, the data of Experiment $2 \mathrm{~B}$ show that subjects were impeded in repeating the same response if the imperative signal on the next trial appeared at the same location as in the previous trial, regardless of its modality.

The data are also instructive because the inhibition of return effect was most evident in the case when subjects were not given a preparatory signal as to the modality of the target. When subjects were given a preparatory signal, the other sequential effects emerged most clearly. In the absence of the preparatory signals, task performance seemed to most clearly reflect the operation of an orienting mechanism concerned with focusing attention on particular spatial locations. By this view, the inhibition of return effect, at least in part, reflects difficulties in reorienting attention to the most recently stimulated spatial location (see Terry et al., 1994). Such difficulties in reorienting attention were greatly diminished in the case where subjects were cued to expect a particular modality.

If it is taken that inhibition of return is the prevalent effect in the basic location judgment tasks used here, because subjects had to attend to a particular location in order to make a response, then it seems that the preparatory signals did play a critical role even at the short SOA. ${ }^{3}$ The presence of the preparatory signal allowed subjects to overcome the natural tendency to inhibit orienting to recently stimulated spatial locations. However, the preparatory signals were not completely effective in this regard given that subjects tended to err when the visual target reappeared on the same side of the space as had the previous target (in Experiment 1). Even when subjects were presented with preparatory signals and made responses to visual targets, they were less accurate when the prime and target occurred at the same location than when the target and prime occurred at different locations.

No such effect was witnessed in the data on auditory target trials. Such a pattern of performance is further evidence that inhibition of return effects are generally more robust in the visual than in the auditory domain (Reuter-
Lorenz et al., 1996). Initially, it was claimed that the lack of evidence for the use of the bypass rule with the visual stimuli fitted with the idea that subjects normally exhibit an attentional bias toward the visual modality (Posner et al., 1976). By this view, subjects attributed equal weight to each response when processing visual targets. However, given the nature of the location judgment task used, a different possibility emerges. It may well have been the case that the bypass rule and inhibition of return were acting in opposition to each other. In contrast, when subjects processed the visual stimuli, these two effects effectively canceled each other out. When subjects processed the auditory stimuli, the weaker inhibition of return effect was overshadowed by compliance with the bypass rule.

\section{Summary and Conclusions}

In summary, the present experiments have revealed a number of quite different sequential effects in CRTs. The stimulus-specific repetition effects and the more general adherence to the bypass rule were evident in the data for the auditory target trials in Experiment 1 . The modality repetition effect was evident in the data for Experiments 1 and $2 \mathrm{~B}$ for both auditory and visual target trials. In addition, inhibition of return was revealed in the data for Experiment $2 \mathrm{~B}$ for both types of target stimuli. The inhibition of return effect has been interpreted in terms of an orienting mechanism responsible for focusing attention at particular spatial locales. Such a tendency to orient attention appeared to be, essentially, overridden by the presentation of the preparatory signals that informed of the relevant stimulus modality. Indeed, when the preparatory signals preceded the target by a reasonably long lag (in Experiment 2A), any influence of the previous trial was abolished. In this sense, therefore, subjects were able to focus attention on the relevant stimulus modality. It is concluded that the presentation of the preparatory signals had two effects. First, they interfered with the spatial-orienting mechanisms to a degree to which only marginal effects of orienting were found with the visual but not the auditory stimuli. Second, they allowed subjects to prepare for (i.e., focus attention on) the relevant stimulus modality given that the preparatory signal-target interval was long enough.

Finally, the experiments have clearly demonstrated that the standard repetition effect and the modality repetition effect reflect different underlying processes. Critically, the modality repetition effect arose in the absence of the repetition effect (in Experiment 1 on the visual target trials and in Experiment 2B for both kinds of targets). Whereas the standard repetition effect may well reflect compliance with the bypass rule (Fletcher \& Rabbitt, 1978; Krueger \& Shapiro, 1981), the modality repetition effect appears to reflect costs in having to switch attention between the auditory and the visual modalities. Indeed, the present set of experiments suggests that there are important differences between the orienting of attention to a particular spatial locale and the focusing of attention to a particular sensory modality. 


\section{REFERENCES}

Bernstein, I. H., Pederson, N. N., \& Schurman, D. L. (1972). Intersensory versus intrasensory contingent information processing. Journal of Experimental Psychology, 94, 156-161.

BERTELSON, P. (1961). Sequential redundancy and speed in a serial twochoice responding task. Quarterly Journal of Experimental Psychology, 13, 90-102.

BERTELSON, P. (1965). Serial choice reaction-time as a function of response versus signal-and-response repetition. Nature, 206, 217-218.

FLetcher, B., \& RabBitT, P. M. A. (1978). The changing pattern of perceptual analytic strategies and response selection with practice in a two-choice reaction time task. Quarterly Journal of Experimental Psychology, 30, 417-427.

Hannes, M., Sutton, S., \& Zubin, J. (1968). Reaction time: Stimulus uncertainty with response certainty. Journal of General Psychology, 78, 165-181.

KRISTOFFERSON, M. W. (1967). Shifting attention between modalities: A comparison of schizophrenics and normals. Journal of Abnormal Psychology, 72, 388-394.

KruEger, L. E., \& SHAPIRo, R. G. (1981). Intertrial effects of samedifferent judgments. Quarterly Journal of Experimental Psychology, 33A, 241-265.

LABERGE, D. (1973). Identification of two components of the time to switch attention: A test of a serial and a parallel model of attention. In S. Kornblum (Ed.), Attention and performance IV (pp. 71-85). New York: Academic Press.

Pashler, H., \& Baylis, G. (1991). Procedural learning: 2. Intertrial repetition effects in speeded-choice tasks. Journal of Experimental Psychology: Learning, Memory, \& Cognition, 17, 33-48.

PoSNER, M. I. (1978). Chronometric explorations of mind. Oxford: Oxford University Press.

PoSNER, M. I., \& COHEN, Y. (1984). Components of visual orienting. In H. Bouma \& D. G. Bouwhuis (Eds.), Attention and performance X: Control of language processes (pp. 531-556). Hillsdale, NJ: Erlbaum.

Posner, M. I., NisSEN, M. J., \& KI EIN, R. M. (1976). Visual dominance: An information-processing account of its origins and significance. Psychological Review, 83, 157-171.

RABBITT, P. M. A. (1968). Repetition effects and signal classification strategies in serial choice-response tasks. Quarterly Journal of Experimental Psychology, 20, 232-240.

RABBITT, P. M. A. (1992). Many happy repetitions: A celebration of the "Bertelson Repetition Effect" 1961-1991. In J. Alegria, D. Holender, J. Junca, \& M. Radeau (Eds.), Analytic approaches to human cognition (pp. 313-330). Amsterdam: Elsevier.

Reuter-Lorenz, P. A., JHA, A. P., \& Rosenquist, J. N. (1996). What is inhibited in inhibition of return? Journal of Experimental Psychology: Human Perception \& Performance, 22, 367-378.

Rogers, R. D., \& MONSELL, S. (1995). Costs of a predictable switch between simple cognitive tasks. Journal of Experimental Psychology: General, 124, 207-231.

SMITH, M. C. (1968). Repetition effect and short-term memory. Journal of Experimental Psychology, 77, 435-439.

SPENCE, C., \& DrIVER, J. (1997). On measuring selective attention to an expected sensory modality. Perception \& Psychophysics, 59, 389-403.
Sutton, S., Hakerem, G., Zubin, J., \& Portnoy, H. (1961). The effect of shift of sensory modality on serial reaction-time: A comparison of schizophrenics and normals. Journal of Abnormal Psychology, 72, 224-234.

Sutton, S., \& ZUbin, J. (1964). Effect of sequence on reaction time in schizophrenia. In A. T. Welford \& J. E. Birren (Eds.), Behavior, ageing and the nervous system (pp. 1-37). Springfield, IL: Thomas.

TANAKA, Y., \& SHImojo, S. (1996). Location vs. feature: Reaction time reveals dissociation between two visual functions. Vision Research, 36, 2125-2140.

Terry, K. M., Valdes, L. A., \& NeILl, W. T. (1994). Does “inhibition of return" occur in discrimination tasks? Perception \& Psychophysics, 55, 279-286.

UMiltà, C., SNyder, C., \& SNyder, M. (1972). Repetition effect as a function of event uncertainty, response-stimulus interval and rank order of the event. Journal of Experimental Psychology, 93, 320-326.

WINER, B. J. (1971). Statistical principles in experimental design (2nd ed.). New York: McGraw-Hill.

\section{NOTES}

1. It is true that response repetition and location repetition are confounded in this design. That is, the same response was required whenever a stimulus fell at a particular location. In this sense, therefore, response repetition and "stimulus repetition" are confounded. However, it will be shown that when the data from Experiments 1 and $2 \mathrm{~B}$ are compared, there appear to be good grounds for arguing that effects due to response repetition (found in Experiment 1) are quite different from those due to location repetition (found in Experiment 2B).

2 . In contrast with Experiment 1, the duration of the visual feedback was set at 300 (vs. 500) msec and the blank interval was set at 200 (vs. $500) \mathrm{msec}$. The intention was to have the blank interval set at $260 \mathrm{msec}$ rather than $200 \mathrm{msec}$, but due to an oversight, this change to the software was not saved. This meant that in Experiment 1, the response stimulus interval (RSI) after a correct response was $1,560 \mathrm{msec}$, and here it was $1,500 \mathrm{msec}$. After an incorrect response, the interval was $1,960 \mathrm{msec}$ in Experiment $\mathrm{l}$ and here it was $1,900 \mathrm{msec}$. As before, though, no scores from target trials that immediately followed an incorrect response to the associated prime were included in the analysis. Even if it is assumed that sequential effects do dissipate with increases in RSI (see Umiltà, Snyder, \& Snyder, 1972), the slight difference in RSI across the experiments suggests that if anything, larger sequential effects should obtain under the present conditions than those used previously.

3. Indeed, in a pilot test exactly the same pattern of effects found in Experiment $2 \mathrm{~B}$ arose when the timing parameters used in Experiment 1 were used, but, again, the preparatory signals were no longer presented. Even when the interval between the offset of the fixation cross and the onset of target was reduced to $60 \mathrm{msec}$, the same modality-switching costs and inhibition of return effects were found.

(Manuscript received July 25, 1997; revision accepted for publication February 26, 1998.) 